\title{
Investigating Decision Making Processes in Distributed Development Teams: Findings of a Comparative Empirical Study
}

\author{
Ban Al-Ani and David Redmiles \\ Donald Bren School of Information and Computer Sciences, University of California, Irvine \\ 444 Computer Science Building \\ Irvine, CA 92697-3440 USA \\ $+1(949)$ 824-2776 \\ \{balanilredmiles\}@ics.uci.edu
}

\begin{abstract}
In this paper, we report the findings of an empirical investigation into decision making processes (DMPes) in distributed teams. We conducted the study in a Fortune 500 organization with a total of sixteen respondents who participated in an hour-long interview. Our analysis of the DMP reported by our respondents suggests that there is a strong organizational culture that supersedes local and individual culture. We also found that while decisions are predominately made in consultation with team members, the leader is usually the decision maker regardless of team distribution or size. The role the leader plays in the DMP implies that greater consideration should be given to assigning a team leader and also the DMP in place. It is also significant that we did not find any evidence of support mechanisms to the DMP leading us to conclude that this possibility needs to be explored by the organization. Our data also suggests that the $D M P$ is chosen ad-hoc and is not explicitly articulated in this organization, which may benefit from documentation.
\end{abstract}

\section{Introduction}

The development process consists of a myriad of activities and requires making decisions throughout at the individual, team, and organizational levels. Developers, at the individual level, typically need to decide on how to design a module and which types of tests to run. Decisions affecting all members of a distributed team will also typically need to be made regarding the milestones and timelines at the team level. Finally, at the organizational level, decisions are also made in terms of team formation and roles.
These levels and the decisions are examples of some the issues that are typically encountered during development. They need to be resolved when they arise in development teams in general but become even more challenging in distributed teams.

We sought to investigate the decision-making processes (DMPes) that are adopted by distributed teams and gain an understanding of these processes. Moreover, we sought to investigate the roles that the leader, culture and team distribution play in the DMP. We identified three distinct DMPes from descriptions of the process employed i.e. consultation, consensus and autocratic. Our data suggests that ultimately it falls on the leader to make the decisions mostly through consultation but sometimes through consensus. Finally, we found that the organization's culture typically overrode other cultures that existed within distributed teams, which implies that team distribution played a minimal role in the DMP. Interestingly, despite respondents' acknowledging the strength of organizational culture their description of the DMP was reflective rather than based on an explicitly defined process leading us to conclude that the process was emergent rather than predefined.

There is an expectation that decision-making in distributed teams is tightly coupled with the characteristics typically found in such teams. For example, Herbsleb and Grinter [7] found that communication frequency contributes to the inefficiency of distributed teams thus decisions must often be made quickly with the limited information available. They also found that developers are more inclined to take the risk that problems will not arise. They report that this can lead to developers being unaware of the outcomes of the DMP even if it affects them because they did not check.

Our findings extend existing knowledge on decision making, especially in the area of leadership, 
some of which is discussed in the following section. This discussion is followed by a description of our research approach and the details of our findings. We also discuss the threats to research validity and the implications of our findings in the final two sections.

\section{Research context}

Our review of literature led us to identify some of the variables which can play a significant role in a decision-making process (DMP) adopted by a distributed team. We consider these variables to be highly interdependent. They include: leadership style, culture and team distribution. While other variables can exist we chose to investigate these in our empirical study. We found that leadership is a key variable in decision-making but also found that it is usually ignored in information systems' literature while the recognition of its significance at both the individual and collective levels is evident $[21,2,13]$.

We also conclude that a leader will typically adopts a leadership style that is either goal oriented and thus directed towards achieving tasks or emotion oriented creating a collaborative emotive environment. Every leadership definition we reviewed fell into this pattern of categorization. For example, one categorization of leadership is provided by Zhang et al, which includes participative leadership and directive leadership [21]. In their view, participative leadership is the equalization of power and the sharing of problem solving with followers by consulting them before making a decision. Thus this leadership style is more focused on emotional commitment from the participants who play a major role in team efforts. They define directive leadership as a leadership which provides and seeks compliance with directions for accomplishing a problem solving task and is therefore can be considered activity focused to achieve goals. We conclude that they consider participative leadership and directive leadership parallel to transformational leadership and transactional leadership respectively.

Other categorizations include those provided by Cogburn et al's [2] who refer again to two categories: task-focused leadership and relationship-focused leadership. Task-focused leadership is a direct leadership focused primarily on achieving the task and thus is typically characterized by dominance behavior. They found that relationship-focused leadership is indirect and typically focuses on improving team cohesion, often associated with cooperative behavior, such as democratic decision- making. Their review of literature led them to conclude that each type of leadership is effective in certain contexts. For example, relationship-focused leadership is often considered advantageous and may even be more effective than task-focused leadership especially amongst mixed gender teams on unstructured tasks. Yet, they state that task-focused leadership is considered more effective than relationship-focused leadership overall. These definitions are also inline with the general goal and emotion focused leadership styles identified by Zhang et al and others.

Cultural variables can also come into play during the DMP. Researchers generally find that a culture can be either individualistic or collectivist. Zhang et al base their definition of these terms on Hofstede's study [21, 8]. They state that in individualistic cultures, the interest of the individual prevails over that of the team; the ties between individuals are loose. Collectivism pertains to societies in which people are integrated into strong, cohesive teams. A highly collectivist culture expects its people to rely upon and give allegiance to the various teams to which they belong. Their examples of collectivist cultures include Japan, South Korea, and Arabspeaking countries whereas the USA, Britain, and Canada are considered highly individualistic.

Culture can also be discussed in terms of western and non-western. In such cases we concluded researchers typically reported that in western societies, decisions are based on consensus or consultation. In cultures with greater hierarchies, e.g. eastern cultures, decisions are usually made by a person who is higher in the hierarchy. Culture can also determine what inference and decision procedures they use [13].

Organizational culture is also an issue that can impact the DMP. For example, we found reference to a "high power-distance culture in which organizations run with a command and control model, i.e., management making the decisions and developers carrying out instructions" in Zhang et al's report [21].

Decisions are made at either an individual or collective level throughout the development process. Thus culture can influence the DMP either because of the individual's background and/or the dominant culture within the Organization or the local culture dominant in the location of the organization's site. At an individual level, developers typically tend to anchor their decisions on available information adjusting their decision making process as new information becomes available [10]. Researchers typically find that expert decision makers usually do 
not make exhaustive calculations or comparisons of alternative solutions [11]. Developers typically need to make decisions about the internals of each module, decided to develop the code manually based on the design agreements, examining each change request and deciding whether, how, when, and by whom it should be fixed, etc [7].

\section{Research method}

We conducted our research in a Fortune 500 organization that has multiple sites distributed worldwide. The organization is considered a leader in computer products design and development. Employees involved in the development of products were invited to participate in the research via email and were informed of the study interview questions. Interview questions focused primarily on task allocation, communication, leadership and the exchange of ideas in teams.

Sixteen employees responded to the invitation and consequently participated in the study. We interviewed respondents utilizing a set of open-ended and close-ended questions. Respondents were encouraged to elaborate their answers and choices regardless of the type of question. Interviews lasted for an hour, on average, and were later transcribed and tabulated.

An analysis of the background information provided by the respondents revealed that their experience in the development domain ranged from 3 to 45 years. We calculated that respondents had a mean of 19.3 years experience with both collocated and distributed projects. We also found that respondents mentioned a total of 26 different sites and that the distributed teams were located on 4 different sites on average.

We asked respondents to answer all questions within the context of two of the most recent projects they had participated in. We asked them to choose a project where all team members were collocated (Team A) and another where one or more members were in a remote location (Team B). We made this request for several reasons. First, we wanted respondents to answer question with regards to a specific project to help them recall their experience within a single instance and thus give us a deeper understanding of the context of their answers [18]. Second, we also asked them to focus on their most recent project to increase the accuracy of their recollection. Finally, we asked them to describe their experience within the context of two different types of teams to provide both the respondents and the researchers with a point of reference for their experiences e.g. better, more efficient...etc.

Respondents also provided abstract descriptions of the projects as all respondents were involved in the development process in one capacity or another. We found that the projects were diverse in type and in deliverables. Projects typically fell into one of three categories, namely: platform, process or product development. Platform development projects consisted of developing software, developing hardware, engineering of, integration of, testing of and manufacturing of platforms. Process development or enhancement refers to projects concerned with developing, optimizing, and documenting, evaluating process or consolidating existing processes. Finally, product development category included the development of software, hardware, engineering of, integration of, testing of and manufacturing of computer based products. Project deliverables were either a new release of an existing product, a product that is new to the organization, or an innovative product that is being developed for the first time (new to the organization and the market). Details of the type of projects and their deliverables are reported elsewhere [1]. We did not find any correlation between the projects and the DMP thus we will not include these details in this report.

\section{Research findings}

We sought to investigate three specific research questions, namely:

1. What roles does the leader play in the DMP?

2. What roles does culture play in the DMP?

3. What role does distribution play in the DMP?

We investigated RQ 1 by asking the question directly, whereas we chose to leave it to the respondents to describe culture and the role distribution plays on the DMP indirectly (research questions RQ 2 and RQ 3). We did this by asking questions regarding distribution of team members and locality of the leader in addition to questions regarding the characteristics a leader should have and the characteristics the leader did actually have in both distributed and collocated teams. Background data provided by the respondents also provided information regarding team distribution and the diversity of culture. We later investigated if a relationship existed between culture and team distribution. The results of investigating these variables are presented in the following sections. 


\subsection{General findings regarding the DMP}

The study revealed that both collocated and distributed teams have nearly the same distribution of both the decision maker and the DMP. The DMP questions were generally opened-ended and the different types of DMPes categories emerged as a result of our analysis of respondents' descriptions of these processes. It should also be noted that some respondents reported that more than DMP could be used to suit the different context of decision. They were asked to describe the most dominant.

We found that their descriptions typically fell into one of three categories, namely: consultation, consensus and autocratic. The first of these three processes, consultation, refers to a process in which the leader made a decision after consulting with selected or all team members. The members are consulted either because they are considered experts in the domain in which a decision has to be made or they will be affected by the outcomes of the decision. Decision making through consensus refers to a process wherein an issue was discussed by selected or all team members until everyone agreed on a single decision. Respondents reported that reaching a unanimous consensus was not always possible, in which case members who disagreed with the majority would still accept the decision. The expression means that consensus is not necessary, nor technically present when adopting this DMP. The general organizational policy is described by one participant as follows:

"If people don't agree they're not real shy about saying they don't agree but the group has to kind of come to a decision and we all know how to do it. We all have that concept of [accepting while differing]. Ok, I [differ], I've said my piece but I will go and do what the group wants me to do. That's a standard kind of thing. If you get into the, I'm a manager and you will have to do what I say then that doesn't play very well in this org. The individual contributor is just as likely to decide well I'm going to do what I think is the right thing anyways and go off and off and do it or go around that persons head talk to someone else."

The statement suggests that this policy leads to a certain organizational culture, which will be investigated further in an oncoming section.

Finally, an autocratic DMP refers to a DMP where the leader states a decision without considering the opinions of any of the team members. These were the three definitions that emerged from our discussions with the respondents during the study interviews.
A summary of the decision maker and the type of decision making process for the collocated and distributed teams is presented in Tables 1 and 2 respectively. We present both to enable a comparison between the DMPes in each type of team. A comparison can help us determine whether the DMPes are specific to distributed teams in the organization or whether our findings are consistent with teams in general in this organization.

Table 1: Summary of decision maker (DM) and process adopted in collocated teams derived from study data.

\begin{tabular}{|c|c|c|c|}
\hline DM & Consultation & Consensus & Autocratic \\
\hline Leader & 10 & 1 & 1 \\
\hline Team & 2 & 2 & 0 \\
\hline None & 0 & 0 & 0 \\
\hline
\end{tabular}

The data in Table 1 demonstrates that while decisions were more often carried out through a process of consultation with the team, the decision maker was usually the leader in collocated teams. The consultation and consensus DMP are democratic processes offering all team members an opportunity to influence what happens in the project in addition to what happens to and in the team. Thus we conclude that the leadership style is predominately participative with regards to decision making, in collocated teams in this organization.

A review of Table 2 also leads to conclude that consultation with the leader as the decision maker is also the prevalent DMP in distributed teams and leads us to conclude that a participative leadership style is dominant in this organization. The data presented in these two tables also implies that there are minimal differences in DMP in collocated and distributed teams in this organization.

Table 2: Summary of decision maker and method for distributed teams derived from study data.

\begin{tabular}{|c|c|c|c|}
\hline DM & Consultation & Consensus & Autocratic \\
\hline Leader & 8 & 1 & 1 \\
\hline Team & 3 & 2 & 0 \\
\hline None & 1 & 0 & 0 \\
\hline
\end{tabular}

We found that the leader typically determined the kind of decision making process adopted yet we found that there was no correlation between the type of power a leader exerted and the DMP. We also find that in the majority of the cases (almost all), decision making is a team activity as opposed to an individual process which can lead to the assumption that culture and team distribution plays a role in the DMP adopted in distributed teams. However, we did not find indications that culture and distribution played a role. This finding together with the realization that our findings were applicable across both types of teams implies that organizational cultural dominated 
the DMP rather than regional or individual culture or the distribution of the team. Furthermore, the lack of correlation between these variables and the DMP implemented led us to conclude that the process was adopted ad-hoc rather than as a result of a study of these variables.

We will present and discuss the findings of our research with regards to each of our research questions (RQ 1, RQ 2 and RQ 3) further in the oncoming sections. Thus we will discuss the role the leader, culture and distribution play in the DMP.

\subsection{The role of the leader in the DMP}

Our data implies that participative leadership style was dominant during the DMP. We defined the term leadership to respondents as the process through which one member of the team (its leader) "influences other team members towards attainment of specific team goals". This definition implies that any team member can exercise leadership and influence over peers, meaning that the existence of a team without leadership is not possible, even though it may not have a formal leader. It also suggests that the leader is not necessarily officially appointed by employees higher up in the development hierarchy and gives study respondents the opportunity to identify who played the role of the leader in their team; we found it was not necessarily the manager.

Respondents were shown a scale which consisted of seven levels from the most negative to the most positive personal attributes and leadership styles. The scale was adapted from Pennington's scale, which was derived from literature $[14,3,4,5]$.

Personal attributes include pleasant, friendly, warm, interesting, efficient and cooperative were listed in Pennington's scale and influenced respondent's discussions [14]. Respondents were also encouraged to provide rationale for their rating and give examples. In one such instance, the respondent checked both sides of the spectrum. When asked why the respondent stated that it was to indicate that she (the leader) was positive when it suited her (with her superiors) but generally negative to her own team members:

"...She was pleasant to outside people not to her own team members. Example, I proposed another way of doing something to the approach she had adopted (but not necessarily proposed) and was harangued e.g. "who the hell do you think you are...etc". People were thus actively discouraged from giving feedback ideas input it was that way because she said if it wasn't that way then should be because she said it. Meetings were deathly quiet with a lot of tension. Efficient only because the talk of the town. She was efficient at what she was willing to take on. Good at presentations. Not good with peers or less. When she left the tension lifted and there was a free exchange of ideas. People were more willing to step outside the box, the atmosphere changed people started to joke. It got fun and the communication started to ease up and also became more effective were no longer a project team on the hot seat."

This is an extreme example of a complex leader and also a rare one. It demonstrates the important role that the leader plays in both the exchange of ideas and the internal decision-making process that was adopted collectively without explicit discussion. Others reported a more positive experience with their leader, which is generally more reflective of the typical responses given and also gave us insight as to why there was no correlation between leadership and decision making.

Questions regarding leadership criteria were openended and respondents were asked to describe their leader. Questions regarding the type of power exerted by the leader to influence team members to follow decisions were closed-ended. An analysis of their description revealed that there was no discernable pattern of leadership. This may be because there does not seem to be any well-defined or widely acknowledged criteria for choosing a leader when respondents were asked to rate the leader. Considering the consultative DMP that is generally adopted leads us to suggest that the participative or relationship leadership style is dominant in this organization. Other researchers have concluded that this leadership style is particularly effective with mixed gender teams [2]. Our data implies that this style is also effective in distributed teams given the general success of the projects at the time of conducting the study. It was not possible to return and investigate the conclusion of the project and conducting a retrospective study of practices with the benefit of hindsight.

The types of power a leader could have were also discussed during the interview. Respondents were all shown the first two columns of Table 3, and asked to identify the type of power their leader had during development in both collocated and distributed teams. The table is extracted from Pennington who derived it from existing literature $[14,6,16]$. A table listing the six types of power and a definition of each type (as in Table 3) in addition to an example of each type of power was presented to each respondent. 
Table 3: The six types of power Pennington [14] derived from French and Raven [6] and Raven [16] in addition to study respondents' description of leadership power.

\begin{tabular}{|c|c|c|c|}
\hline Type of Power & Description & Collocated Team & Distributed Team \\
\hline Reward power & $\begin{array}{l}\text { Ability of the leader to provide what others want or } \\
\text { remove what they dislike or do want. }\end{array}$ & 6 & 2 \\
\hline $\begin{array}{l}\text { Referent } \\
\text { power }\end{array}$ & $\begin{array}{l}\text { Respect with which the leader is held by team } \\
\text { members and ability to create common sense of } \\
\text { identity. }\end{array}$ & 9 & 5 \\
\hline $\begin{array}{l}\text { Informational } \\
\text { power }\end{array}$ & $\begin{array}{l}\text { Use of information, which may be privileged to the } \\
\text { leader, in a logical way to present compelling } \\
\text { argument. }\end{array}$ & 8 & 7 \\
\hline $\begin{array}{l}\text { Legitimate } \\
\text { power }\end{array}$ & $\begin{array}{l}\text { Team members accept rules and norms of the leader } \\
\text { and regard leader as properly occupying the position. }\end{array}$ & 8 & 5 \\
\hline Expert power & $\begin{array}{l}\text { Leader perceived and regarded as having superior } \\
\text { knowledge and/or specialist in a field. }\end{array}$ & 6 & 7 \\
\hline $\begin{array}{l}\text { Coercive } \\
\text { power }\end{array}$ & $\begin{array}{l}\text { Leader has the ability to threaten and punish others to } \\
\text { get his or her own way. }\end{array}$ & 2 & 1 \\
\hline
\end{tabular}

Table 3 includes the information provided by our respondents regarding the type of power they identified. As stated previously, we asked each respondent to answer both questions within the context of their most recent experience in their collocated and distributed teams to provide both the respondents and researchers' points of comparison. We wanted to explore whether our findings were due to the nature of the team (i.e. distributed vs. collocated) or other factors (e.g. organizational culture). In this instance we found that the leader in both distributed teams and collocated teams demonstrated similarities in the type of power our respondents identified. We also found that leaders in both types of teams typically demonstrated more than one type of power. Furthermore, we found that collocated teams had more variations in the combination of power types demonstrated by the leader. Thus our data implies that collocated team leaders have more opportunity to demonstrate different types of power to influence their teams.

Respondents often provided rationale as to why they felt the leader had the power(s) identified. For example one respondent explained the evidence of different power types demonstrated by the leader of his distributed team:

"...it started out as informational because he tended to grab hold, he wasn't really a leader and he just started gathering all the information and had the slides and everything so he knew more than anyone else. Then because he did he got anointed like ok you really run this group. and the boss, the leader of the org turned to him because he had more data than the rest of the people, so he went from informational to legitimate. I think there was a transition there."

This statement and others like it imply that leaders typically start their leadership with one type of power that generally evolves to include other types over time. Respondents' statements also typically imply that in this organization there is a culture of empowerment, which is inline with our findings that the DMP is independent of leadership style.

Finally we found no significant difference in the DMP adopted in collocated teams where the team leader was always collocated with team members and the DMP adopted by the team leader in a distributed team where the leader was not necessarily collocated with the majority of the distributed team member and a leader who was never collocated with all team members. Thus we conclude that the location of the team leader was not a factor that affected the DMP in this organization.

\subsection{The role of culture}

We found that respondents typically referred to either organizational culture or regional culture throughout our interviews within the context of the various aspects of distributed collaboration that we discussed. Our data implies that organizational culture generally dominated team practices, thus we found that regional culture did not have a discernable impact on the DMP. The organizational culture is evident in the policy to differ but still accept decisions, for example. This policy implies that the organizational culture demonstrates both collectivist and individualistic characteristics. It is collectivist in nature in that the team is required to collectively agree to commit to a decision through the consensus DMP. If an individual within the team disagrees then the individual will still commit to a decision made by the team. Our data suggest that individualistic and hierarchal behavior in such a context by a leader can cause team members to exhibit individualistic behavior also.

The seemingly hybrid nature of the organization's culture is also evident in the consultative DMP that is prevalent in our study. Here we found that while the leader consults with the team members, the leader is typically the decision maker. Thus the leader, 
perceived as being higher in the hierarchy than other team members demonstrates the characteristics of an individualistic culture yet also demonstrates some characteristics of a collectivist culture by consulting with other team members. The blend of cultural characteristics may be the result of the size of the organization which is distributed world-wide, the influence of multiple cultures within a single site or a combination of the two. Further evidence of the individualistic nature of the organization's culture can be found in another respondent's description of its impact on the DMP:

"...here [in the organization] there is an underlying [organization named] culture that's much different than other organizations. There [are] things that spark the confrontation, that's not pleasant, it's not expected to be pleasant, it's expected that if you have an issue you need to confront the person, whether or not they're pleasant or not it's expected, it's a much different culture than I've seen in other companies I've worked for. So there is an expectation in our Organization in our core values that they expect you to do that."

This statement clearly highlights the general feeling regarding the prevalence of organizational culture and the dominance of this culture within the organization's sites regardless of locality. This finding is in contrast to answers regarding communication, where we found respondents felt that culture did play a role in communication. For example, one respondent described the communication model as being ineffective because the large number of developers involved led also to diversity in cultures. Furthermore, the respondent's description also touches upon the DMP, the challenges encountered when it is applied across sites and the actions typically taken to overcome these challenges.

"[the model is] ... not effective as A [collocated team] because they [the developers] are new and cultural[ly] they are more conservative. Within the organization in that country they don't take risk it is the culture there. They don't want to stand up and be an individual they want to merge as a team and be lost in the team rather than be an individual. We have a lot of problems communicating. They don't say it in the meeting. If asked if they agree, for example, I only hear silence so I don't know if it's yes or no. So it's not the model but the culture their ability to respond to communications which is not meeting expectancy. That's why we want to bring their other members here to integrate them. I asked our managers/leaders to leave when they came face- to-face so I can talk to them directly and spent time to understand why they weren't talking..."

The respondent describes interactions with others from a different, seemingly more collective culture, and their unwillingness to distinguish themselves as individuals. The result is that this respondent feels they fail to communicate and the seeming failure of the consultative approach in such instances. He went on to describe his suspicion that while:

"...Everyone is given the opportunity to state argument and the leader then communicated these to the manner. However I did not have confidence that the people did present arguments I felt that it might be the member followed the manager's lead. I don't know how much they speak. I think the manager takes the leaders. Other times they will follow top-down model where the manager will decide something and inform those in the lower ranks to follow through. It flows down to the foot soldiers"

The respondent's response demonstrates that while the consultative approach was adopted and was challenged by a different local culture; this leader strove to limit the impact of these challenges by both including team members lower in the team hierarchy and addressing the local culture. The leader strove to address local culture by "integrating" team members at the remote site. Integrating remote team members into organizational culture was also referred to by other respondents in contexts other than decision making. The following is another example of just such a reference:

"It was my decision on how to communicate. The first kick off 2 sessions we didn't try to get all the developers. I tried to alternate meetings. So that one meeting the US team go over there the next time they go over to US. Not all but some of them and they go back and integrate."

Both these statements provide examples that demonstrate respondents' awareness of other cultures and the general attitude that the hybrid organizational culture should prevail regardless.

These observations and respondents' statements collectively led us to conclude that there is general expectancy that new team members will assimilate and adopt the organization's culture over time. Thus, the likelihood that this organization exports its culture to sites overseas is high and may be the root cause of our finding that the impact of local culture is minimized as a result of "integration". 


\subsection{The role of team distribution}

We also explored the possibility that the locality of the leader impacted the DMP adopted by the team and whether the majority of the team was collocated with the leader. We found that the leader is predominately located with the majority of the participants when the teams are distributed and was considered an advantage by study respondents. Participants considered it a disadvantage when the leader was located with the minority of team members, regardless of where the respondent was located. Furthermore, our data implies that the respondents overwhelmingly felt that the leader's location did not impact the work distribution when the leader was located with the minority. When the leader was located with the majority or co-located, about $50 \%$ of the respondents felt that the leader's location impacted the work distribution. However, we did not find any correlation between the locality of the leader and the DMP adopted.

The following statement by one respondent gives us insight into how the impact of the leader's locality is minimized in the organization allowing the adoption of a DMP without constraint. The consultative DMP adopted was enabled because of the empowerment of team members and the general organizational culture which encourages participation:

"...people were given an opportunity to voice their ideas and did so despite the time difference. Even the geos [geographically remote members]. For example one member called when it was 1:00 am his time so that he can participate in a meeting during working hours and designated meeting time. This is encouraged in [the organization] and well known by its long term employees. Despite the culture differences, language barriers and differences in time zones ideas/opinions were being expressed and taken up/acknowledged spontaneously because of the type of leadership in place, the confidence of the long term employees in the culture of the organizations which is prevalent in all the organizations' sites global[ly]."

Here, we again benefited from the comparative nature of our study to investigate the accuracy of the conclusions made. As stated previously, we found no discernable difference in the DMP adopted in collocated team, where the majority (if not all) of the members were typically from the US, and distributed teams where team members were usually characterized by cultural diversity. Our data also implies that the number of sites over which the team is distributed and its associated implications (e.g. differences in time zone, culture, languages...etc) had no discernable impact on the DMP. We also found that the distribution pattern (i.e. the majority located with leader vs. minority collocated with leader) did not have a discernable impact on the DMP adopted by a distributed team.

\section{Threat to validity}

We are aware that there are some threats to experimental validity. First, while our study that was conducted across multiple sites, it was conducted within a single organization. Second, the limited number of respondents who participated in the study also threatens the generalizeability of our study.

We also recognize that limiting our data to that gathered during the interview without corroborating our findings with data from other sources (e.g. emails, meetings...etc) can also be considered a threat to study validity. This limitation can introduce bias and inaccuracies that we strove to minimize by focusing on the most recent team experiences. However, these threats do not prevent us from making several significant observations that can extend existing understanding of this domain.

\section{Discussion}

Our review of literature led to our investigating DMP in conjunction with leadership, team distribution and culture. We found a great deal of diversity in leadership styles and while we found no correlation between the power of the leader uses to influence teams member or the leader's personal attributes and the DMP adopted; we did find that a participative leadership style was implied from the DMP adopted.

Respondents were unaware of why leaders were chosen and sometimes stated that it was more a question of availability, yet we also found that the leader played a central role in the DMP. Consequently, the leader who is in control of the DMP may or may not posses the required skills to guide it. These findings also suggests that an effort should be made to allocate a leader in the location of the majority of team members to minimize the challenges that typically arise as a result of a distributed setting, particularly when the consultative or a consensual DMP is adopted. Furthermore, while we did not find the variable that literature led us to anticipate would impact the DMP, our data does imply that a need to investigate whether manipulating 
these variables and the choice of the DMP can lead to more effective decision making in distributed team.

The study also revealed that the DMPes and the effectiveness of each within the various contexts of culture and team distribution require further investigation within the organization. Such an investigation may lead the organization to develop an analytic approach to the choice of DMP and increase the effectiveness of the DMP as we also found a lack of explicitly defined DMPes. Respondents typically needed to reflect on the process and identified it in hindsight, which also indicates that the process should be better documented and distributed in addition to enabling employees to acquire the necessary skills. Alternatively, as no criteria or leadership processes were found to be explicitly defined then these are two items that need to be explored further and addressed.

We could find no links between team distribution and the DMP. We anticipated that the DMP would correlate with either the location of the leader in relation to the majority of the team member and/or the sites and consequently with the culture of the region in which the site was located. Our data revealed no such correlation. However, our data did imply that the organization's culture seems to dominate the DMP. The organization's culture implies that the organization's distribution across multiple-site has led to both the organization exporting the culture of its origin (US) and also absorbing some of the cultural traits of its remote sites. Thus we conclude that the organization's culture is a hybrid of characteristics from these collective cultures. While the regional culture of the organization's origin is more evident, the traits of other cultures may rise closer to the surface as the organization continues its evolution.

\section{Implications}

Our research leads to several implications for the development of automated support tools. The dominance of the consultative and consensual DMPes imply that distributed teams may benefit from some kind of tool to support the DMP. The dominant DMPes identified in our data (consultative and consensual) suggests a web-based decision making tool that will allow developers to structure their interactions into separate dialogues for each issue that they needed to address and thus facilitate the coordination of distributed decision making $[9,12]$.

We found that neither the DMP nor the documentations of its outcomes were explicitly defined within this organization. Documenting the
DMP and its outcome(s) has been identified as key to overcoming misunderstandings that typically arise during distributed decision making [7, 9]. Providing a decision making support tool with the ability to document the DMP and also allow remote members to contribute to it regardless of differences in time zones may improve the efficacy and effectiveness of the DMP adopted.

A tool that provides such support would also need to explore what needs to be documented and what should not be revealed. Documenting too little may mean losing vital decision making rationale, documenting everything may mean overwhelming developers with information [1]. Research has shown individuals do not have a complete understanding of what they need to be mindful of within the organization and it is often only in retrospect that an individual can make sense of symptoms that may lead them to make the correct decisions $[19,20]$. Thus, developing automated support for distributed decision making still requires determining what is to be revealed, when, to whom, and how which are questions we are investigating within different contexts [17].

We found that the organizational culture dominated the DMP and an expectation for new members to assimilate over time. There was also a suggestion that the organizational culture resembled that of the culture of the organization's origin. The organization originated in the US and thus the DMPes we identified reflect the dominance of the egalitarian processes in place in most aspects of US culture. Olson and Olson [13] report that the decision-support systems designed in the United States embody algorithms that fit egalitarian, democratic participation. Their review led them to conclude that while these systems provide anonymity and focus on the task they also tend to ignore any aspect of relationships and obligation. Thus decision support systems designed in the US may prove to be more effective when adopted in organizations which originate in egalitarian locations. However, such systems may prove to be less effective in other organizations and alternatives need to be investigated such that other cultures are also considered.

We should also emphasize that this study reflects the practices of one organization. Thus the implications for potential features of tool support may not be applicable or useful to other organizations in the same domain or different domains. Other organizations and the DMPes in place need to be investigated to identify the features of appropriate decision support mechanisms. 
We also have to consider that a tool may not be adopted even if the tool has been tailored to meet an organization's needs. Introducing a new tool is typically challenging and not necessarily more effective. New tools generally meet with resistance that requires considerable effort to overcome especially if the tool has to be adopted uniformly across all sites to ensure that all members of a distributed team can participate in the team decision making processes. The challenges become more complex when considering collaborations across organizations and the effort required to promote tool acceptance across multiple organizations [15].

Interestingly, we did not find any automated tool was used to support current processes despite asking explicitly about tools. The respondents did not express dissatisfaction with the manner in which the DMP was implemented within the organization. The organization is a leader in its field, which implies that we need to investigate the other mechanisms in place in this organization that support the DMP and enables distributed teams, in particular, to perform. Increasing the effectiveness of existing mechanisms, which may or may not be automated, can lead to an increasingly effective DMP without the overhead of tool development and the process of incorporating the tool into the development process.

\section{Acknowledgements}

The empirical study was supported by a P.E.P grant awarded by the University of Technology, Sydney and by the U.S. National Science Foundation under grants 0534775 and 0808783.

\section{References}

[1] Al-Ani, B., and Edwards, K., (2008). "A Comparative Empirical Study of Communication in Distributed and Collocated Development Teams," IEEE International Conference on Global Software Engineering, Bangalore, India, 17-20 Aug., pp.35-44.

[2] Cogburn, D. L., Zhang, L. and Khothule, M. (2002) 'Going global, locally: The socio-technical influences on performance in distributed collaborative learning teams'. In ACM Research Conference of the South African Institute of Computer Scientists and Information Technologists on Enablement through Technology. September 16-18, 2002. Port Elizabeth, South Africa. Vol. 30, pp. 52-64, South African Institute for Computer Scientists and Information Technologists.

[3] Fiedler, F. E. (1964). A contingency model of leadership effectiveness. In L. Berkowitz (Ed.), Advances in experimental social psychology. New York: Academic Press.
[4] Fiedler, F. E. (1965). Engineer the job to fit the manager. Harvard Business Review, 43, 115-122.

[5] Fiedler, F. E. A theory of leadership effectiveness. New York: McGraw-Hill, 1967.

[6] French, John R., \& Bertram Raven. (1959) "The bases of social power." In: Studies in social power, edited by Dorwin Cartwright. Ann Arbor, MI: University of Michigan Press. pp. 150-167.

[7] Herbsleb J, Grinter R. Architectures, coordination, and distance: Conway's law and beyond. IEEE Software 16(5): 1999, 963-70.

[8] Hofstede, G. (1980), Culture's consequences: International differences in work related values, Thousand Oaks, CA: Sage Publications, Inc.

[9] Holton J.A., Building trust and collaboration in a virtual team, Team Performance Management, Emerald Group Publishing Ltd, 7(3-4), 2001, pp. 3647(12).

[10] Hung, C., Dennis, A., Robert, L. Trust in Virtual Teams: Towards an Integrative Model of Trust Formation. Hawaii international Conference on System Sciences, Track 1, Volume 1 Jan. 5 - 8, 2004.

[11] John D Lee, Katrina A See. Trust in automation: designing for appropriate reliance. by: Human Factors, Vol. 46, No. 1. (2004), pp. 50-80.

[12] Kjeld Schmidt, Liam J. Bannon, Taking CSCW Seriously: Supporting Articulation Work, Computer Supported Cooperative Work, 1(1-2):7-40, 1992.

[13] Olson, J. S. and Olson, G. M. 2003. Culture Surprises in Remote Software Development Teams. Queue 1, 9 (Dec. 2003), 52-59.

[14] Pennington, D. C., (2002). The social psychology of behavior in groups, Psychology Press Ltd.

[15] Powell, A., Piccoli, G. and Ives, B. 2004. Virtual teams: A review of current literature and directions for future research', SIGMIS Database, 35(1), 6-36.

[16] Raven, B. H. (1993). The bases of power: Origins and recent developments. Journal of Social Issues, 49 (Whole no. 4): 227-251.

[17] Redmiles, D., van der Hoek, A., Al-Ani, B. Hildenbrand, T., Quirk, S., Sarma, A., Silva Filho, R., de Souza, C., and Trainer, E., "Continuous Coordination: A New Paradigm to Support Globally Distributed Software Development Projects, Withschaftsinformatik, 49:2007, p. S28-S38.

[18] Sudman, S., Bradburn, N. M., Schwarz, N., Thinking About Answers: The Application of Cognitive Processes to Survey Methodology, Wiley \& Sons, Oct. 1995, chapter 3.

[19] Weick, K. and Sutcliff, K., Mindfulness and the Quality of Organizational Attention, Organization Science 17(4), July-August 2006, pp. 514-524.

[20] Weick, K. and Sutcliff, K., Obtsfeld, D., Organizing and the Process of Sensemaking, Organization Science, 16(4), July-August 2005, pp. 409-421.

[21] Zhang, S., Fjermestad, J., and Tremaine, M. (2005), Leadership Styles in Virtual Team Context: Limitations, Solution and Proposition, Proceedings of the 2005 38th Hawaii International Conference on System Sciences, Hawaii, U.S., pp.11-21. 